\title{
Optimal Stopping and the American Put
}

\author{
S.D. Jacka \\ Department of Statistics \\ University of Warwick \\ Coventry \\ CV4 7AL \\ UK
}

\begin{abstract}
We show that the problem of pricing the American put is equivalent to solving an optimal stopping problem. The optimal stopping problem gives rise to a parabolic free-boundary problem. We show there is a unique solution to this problem which has a lower boundary. We identify an integral equation solved by the boundary and show that it is the unique solution to this equation satisfying certain, natural, additional conditions. The proofs also give a natural decomposition of the price of the American option as the sum of the price of the European option and an 'American premium'.
\end{abstract}

KEYWORDS: AMERICAN OPTION, PUT, OPTIMAL STOPPING, OBSTACLE PROBLEM, FREE-BOUNDARY PROBLEM, MARTINGALE.

\section{$\S 1 \quad$ Introduction}

This paper concerns itself with the problem of pricing the basic American put option. The option confers the right to sell a unit of stock at any time up to the time horizon $T$. We assume that the stock pays no dividends during the lifetime of the option and that the stock price at time $t, X_{t}$, is an exponential Brownian motion:

$$
X_{t}=X_{0} \exp \left(\sigma B_{t}+\left(\mu-1 / 2 \sigma^{2}\right) t\right)
$$

where $\left(B_{t} ; t \geq 0\right)$ is canonical Brownian motion. Thus $X_{t}$ is the unique (strong) solution to the stochastic differential equation:

$$
d X_{t}=\sigma X_{t} d B_{t}+\mu X_{t} d t
$$

with fixed initial value $X_{0}$. We also assume that cash generates interest at a fixed rate $\alpha(>0)$.

This paper establishes, using elementary techniques, the 'well-known' result that the fair price for the option, regarded as a function of the present stock price and the time horizon, is the (essentially) unique solution to a parabolic free-boundary problem; Theorem 4.2.1 (see, for example, McKean (1965) and Van Moerbeke (1976). We establish certain basic properties of the (lower) free boundary $b(t)$; and show that $b(t)$ is the unique left-continuous solution of a convolution-type integral equation; Theorem 4.2.2. 
The result that the option price is the solution to an optimal stopping problem — generalising the Black-Scholes option pricing formula (Black and Scholes (1973)) is proved in Karatzas (1987) but we give a summary in section 3 for the sake of completeness. For a summary of the hedging portfolio technique for pricing options see the excellent introduction in Harrison and Pliska (1981). The main contribution of this paper is the verification of the essential uniqueness of the solution to the free boundary problem and the identification of the integral equation satisfied by the stopping boundary, which may prove numerically more tractable than the original free boundary problem.

Similar results are presented in Kim (1990) and Carr,Jarrow and Myneni (1990): Kim (1990) discusses numerical approaches to solving the integral equation for the stopping boundary, Carr, Jarrow and Myneni (1990) discuss analytic approximations and comparative statics.

The techniques developed in this paper are applied to more general optimal stopping problems in Jacka and Lynn (1990).

\section{$\S 2$ The optimal stopping problem}

2.1 In this section we shall consider the problem of optimally stopping $e^{-\alpha t} X_{t}$ before time $T$ - that is, to find

$$
f(x, t) \stackrel{\text { def }}{=} \sup _{\tau \leq t} \mathbb{E}_{x}\left[e^{-\alpha \tau} X_{\tau}\right]
$$

We work throughout this section with the martingale measure $\mathbb{P}$ (the measure $\mathbb{P}$ is the one which makes the discounted stock price $-e^{-\alpha t} X_{t}-$ a martingale). We shall show that the form of the solution is to stop (i.e. exercise the option), the first time that $X_{s}$, the price of the underlying stock, falls below the moving boundary $b(T-s)(T$ is, as before, the original time to expiry of the option), we shall also deduce some properties of $b(\cdot)$ as we proceed.

Under the measure $\mathbb{P}, X_{t}$ satisfies the SDE:

$$
X_{t}(x)=x+\int_{0}^{t} \sigma X_{s}(x) d B_{s}+\int_{0}^{t} \alpha X_{s}(x) d s,
$$

where $B$ is a $\mathbb{P}$-Brownian motion, so that

$$
X_{t}(x)=x \exp \left(\sigma B_{t}+\left(\alpha-1 / 2 \sigma^{2}\right) t\right)
$$

Theorem 2.1.1 The optimal payoff from the stopping problem is a function only of the present price of the stock and of the time to expiry of the option:

$$
v_{T}=f\left(X_{0}, T\right)
$$

the function $f$ is continuous; $f(x, t) \geq(c-x)^{+}$; and the optimal stopping time $\tau$ is given by

$$
\tau=\inf \left\{s: f\left(X_{s}, T-s\right)=\left(c-X_{s}\right)^{+}\right\}
$$


Proof: The result follows immediately from Theorems 3.1.8 and 3.1.10 of Krylov (1980)

It is immediate from Theorem 2.1.1 that the optimal stopping time $\tau$ is given by

$$
\tau=\inf \left\{t \geq 0:\left(X_{t}, T-t\right) \notin D\right\}
$$

where $D$, given by

$$
D=\left\{(x, t) \in \mathbb{R}_{+} \times \mathbb{R}_{+}: f(x, t)>g(x) \equiv(c-x)^{+}\right\},
$$

is the continuation region for the stopping problem.

Lemma 2.1.2: The payoff $f(x, t)>0$ for all $x \geq 0, t>0$.

Proof: Note that $g(x)>0$ for all $x<c$; for $x \geq c$ and $t>0$

$$
f(x, t) \geq c / 2 \mathbb{E}_{x} e^{-\alpha \tau} c / 2 I\left(\tau_{c / 2}<t\right)>0
$$

where

$$
\tau_{c / 2}=\inf \left\{s \geq 0: X_{s} \leq c / 2\right\} \wedge t
$$

We can now establish the crucial result:

Proposition 2.1.3: For each $t>0$, the $t$ section of $D$ is given by

$$
D_{t} \equiv\{x:(x, t) \in D\}=(b(t), \infty),
$$

for some $b(t)$ satisfying $c>b(t)>0$.

Proof: All we need to do is to prove that $\left(x \in D_{t}\right) \Rightarrow\left(y \in D_{t}\right)$ for any $y>x$, since 0 is clearly not in $D_{t}$. To do this we use a pathwise comparison result based on (2.1.1). Suppose $x \in D_{t}, y>x$, and let $\tau=\inf \left\{s \geq 0:\left(X_{s}(x), T-s\right) \notin D\right\}(\tau$ is the optimal stopping time for $X_{s}(x)$ ); then

$$
\begin{aligned}
& f(y, t)-f(x, t) \\
= & f(y, t)-\mathbb{E} e^{-\alpha \tau}\left(c-X_{\tau}(x)\right)^{+} \quad(\text { since } \tau \text { is optimal for } X .(x)) \\
\geq & \mathbb{E}\left(e^{-\alpha \tau}\left\{\left(c-X_{\tau}(y)\right)^{+}-\left(c-X_{\tau}(x)\right)^{+}\right\}\right) \quad(\text { since } \tau \text { is feasible for } X .(y)) \\
= & \mathbb{E}\left[e^{-\alpha \tau}\left\{\left(c-X_{\tau}(y)\right)-\left(c-X_{\tau}(x)\right)\right\}\right] \\
& + \\
& \mathbb{E}\left[e^{-\alpha \tau}\left\{\left(c-X_{\tau}(y)\right)^{-}-\left(c-X_{\tau}(x)\right)^{-}\right\}\right]
\end{aligned}
$$

Now from the pathwise solution given in (2.1.1), $X_{\tau}(y) \geq X_{\tau}(x)$ so that the second term on the right-hand side of (2.1.3) is non-negative so we see that

$$
\begin{aligned}
f(y, t)-f(x, t) & \geq \mathbb{E}\left\{e^{-\alpha \tau}\left(X_{\tau}(x)-X_{\tau}(y)\right)\right\} \\
& =(x-y) \mathbb{E} \exp \left(\sigma B_{\tau}-1 / 2 \sigma^{2} \tau\right) \\
& =x-y
\end{aligned}
$$


since $\exp \left(\sigma B_{t}-1 / 2 \sigma^{2} t\right)$ is a martingale started at 1 and $\tau$ is a bounded stopping time. Thus $f(y, t) \geq(x-y)+f(x, t)$ and so since $f(x, t)>(c-x)^{+}$(by virtue of the fact that $\left.x \in D_{t}\right)$ we see that

$$
f(y, t)>(x-y)+(c-x)^{+} \geq(c-y)
$$

moreover, from Lemma 2.1.2, $f(y, t)>0(\forall t>0)$ so

$$
f(y, t)>(c-y)^{+}
$$

Note that $b(t) \leq c$ for all $t>0$ since if $x>c,(c-x)^{+}=0$ whilst $f(x, t)>0$

2.2 We shall now establish some of the properties of the boundary $b$.

\section{Lemma 2.2.1}

(i) $f(\cdot, t)$ is decreasing for each $t$.

(ii) $f(x, \cdot)$ is increasing for each $x$.

\section{Proof:}

(i) this follows immediately from (2.1.1).

(ii) this follows immediately from the fact that if $\tau$ is admissible for the optimal stopping problem with horizon $t$ it is admissible for the problem with horizon $s$ for any $s \geq t$

Proposition 2.2.2 The boundary $b$ is decreasing in $t$ and bounded above by $c$.

Proof: By lemma 2.2.1, $f(x, \cdot)$ is increasing, so for any $t>0 ; s \geq 0, \varepsilon>0$

$$
\begin{aligned}
f(b(t)+\varepsilon, t+s) & \geq f(b(t)+\varepsilon, t) \\
& >g(b(t)+\varepsilon) \quad\left(\text { since } b(t)+\varepsilon \in D_{t}\right)
\end{aligned}
$$

and so for any $\varepsilon>0, s>0, b(t)+\varepsilon \in D_{t+s}$ thus

$$
b(t+s) \leq b(t)
$$

and $b$ is decreasing.

Finally note that $g$ vanishes on $[c, \infty)$ whilst if $x \geq c, t>0$ then, from Lemma 2.1.2 $f(x, t)>0$ so that $b(t)<c \quad \forall t>0$

We can now discern the limiting behaviour of $b(t)$.

Proposition 2.2.3 Let $(F, b)$ be the unique solution, with $F \in C^{1}$, to

$$
\left.\begin{array}{r}
1 / 2 \sigma^{2} x^{2} F^{\prime \prime}(x)+\alpha x F^{\prime}(x)-\alpha F=0: x \geq b \\
F(x)=c-x: x<b
\end{array}\right\}
$$


then

(i) $b=\frac{2 \delta}{1+2 \delta} c$

$F(x)=\left\{\begin{array}{l}(c-b)\left(\frac{b}{x}\right)^{2 \delta}: x \geq b \\ c-x: x<b\end{array}\right.$

where $\delta=\alpha / \sigma^{2}$ and $F(x) \equiv \sup _{\tau} \mathbb{E}_{x} e^{-\alpha \tau} g\left(X_{\tau}\right)$

(ii) $\lim _{t \rightarrow \infty} f(x, t)=F(x)$

(iii) $\lim _{t \rightarrow \infty} b(t)=b$

\section{Proof:}

(i) is a well-known result and may be proved directly from Theorem 2.1.1.

(ii) It is clear that $f(x, t) \leq F(x) \forall t$; conversely, let $\tau=\inf \left\{t \geq 0: X_{t} \leq b\right\}$ then $F(x)=\mathbb{E} e^{-\alpha \tau} g\left(X_{\tau}\right)$ and if we define $\tau_{t}=\tau \wedge t$ then for $x>b$

$$
\begin{aligned}
f(x, t) & \geq \mathbb{E}_{x} e^{-\alpha \tau_{t}} g\left(X_{\tau_{t}}\right) \\
& \geq(c-b) \mathbb{E}_{x} e^{-\alpha \tau} I(\tau \leq t) \\
& \stackrel{t}{\longrightarrow}(c-b) \mathbb{E}_{x} e^{-\alpha \tau} \equiv F(x)
\end{aligned}
$$

(since $e^{-\alpha t}$ is a bounded continuous function on $\mathbb{R}_{+}$and $\tau_{t} \stackrel{a . s .}{\longrightarrow} \tau$ ). For $x \leq b$, $f(x, t) \geq g(x)=F(x)$.

(iii) Given $x>b, F(x)>(c-x)^{+}$so, letting $\varepsilon=F(x)-(c-x)^{+}$, if we take $T(\varepsilon)$ such that

$$
F(x)-f(x, t) \leq \varepsilon / 2 \quad \forall t \geq T(\varepsilon)
$$

we see that

$$
f(x, t)>(c-x)^{+}
$$

for $t \geq T(\varepsilon)$ so we conclude that $\lim _{t \rightarrow \infty} b(t) \leq b$. Conversely if $x<b$ then

$$
(c-x)^{+}=F(x) \geq f(x, t)
$$

so $x \leq b(t) \forall t$

Proposition 2.2.4 The boundary b is left-continuous.

Proof: Both $f$ and $g$ are continuous so, since $D=\{(x, t): \quad f-g>0\}, D$ is open, and thus $D^{c}$ is closed. Since $D^{c}$ is closed, if we take a sequence $\left\{t_{n}\right\} \uparrow \uparrow t$ we see that $\left(b\left(t_{n}\right), t_{n}\right) \in D^{c} \quad \forall n$ so that $b(t-) \leq b(t)$ giving us left-continuity of $b$, by virtue of the decreasing nature of $b$

Proposition 2.2.5 The boundary b is right-continuous, and thus, by virtue of Proposition 2.2.4, continuous.

We defer the proof of this until section 2.4. 
2.3 We now establish the relationship between $f$ and a free boundary problem.

Proposition 2.3.1 The payoff function $f$ is the unique solution to the initial value problem:

$$
\left.\begin{array}{l}
\mathcal{L} \phi=0: x>b(t) \\
\phi(b(t), t)=c-b(t): t \geq 0 \\
\phi(x, 0)=(c-x)^{+} \\
\phi(x, t) \stackrel{x \rightarrow \infty}{\longrightarrow} 0 \text { uniformly on compact sets }
\end{array}\right\}
$$

where $\mathcal{L}$ is the parabolic operator

$$
{ }_{1 / 2} \sigma^{2} x^{2} \frac{\partial^{2}}{\partial x^{2}}+\alpha x \frac{\partial}{\partial x}-\alpha-\frac{\partial}{\partial t}
$$

Proof: We first establish that $f$ satisfies (2.3.1). Take $(x, t) \in D$, since $D$ is open we may take an open rectangle $R=\left(x_{1}, x_{2}\right) \times\left(t_{1}, t_{2}\right)$ with $(x, t) \in R \subseteq D$. Now consider the initial value problem

$$
\left.\begin{array}{l}
\mathcal{L} \phi=0:(x, t) \in R \\
\phi(x, t)=f(x, t):(x, t) \in \partial R \backslash\left(x_{1}, x_{2}\right) \times\left\{t_{2}\right\}
\end{array}\right\},
$$

the existence (and uniqueness) of a solution to this initial value problem is given by Theorem 3.6 of Friedman (1975). Let us define

$$
N_{s}^{t}=e^{-\alpha s} \phi\left(X_{s}(x), t-s\right) .
$$

It follows immediately from Itô's formula that $N_{s \wedge \tau}$, where

$$
\tau=\inf \left\{s \geq 0: \quad\left(X_{s}, t-s\right) \notin R\right\}
$$

is a bounded martingale, so that

$$
\phi(x, t)=N_{0}^{t}=\mathbb{E} N_{\tau}^{t}=\mathbb{E} e^{-\alpha \tau} f\left(X_{\tau}, t-\tau\right)=f(x, t)
$$

the last equality following from the fact that $\tau$ is bounded above by the first exit time of $\left(X_{s}(x), t-s\right)$ from $D$.Thus f satisfies $\mathcal{L} f=0$ in $D$. The boundary conditions in (2.3.1) are clearly satisfied by $f$; the fact that $f(x, t) \stackrel{x \rightarrow \infty}{\longrightarrow} 0$ uniformly on compact sets follows from the fact that $f(x, t) \stackrel{x \rightarrow \infty}{\longrightarrow} 0$ for each $t\left(0<f(x, t) \leq c \mathbb{P}\left(\tau_{c}(X .(x)) \leq t\right)\right.$ for $x>c$ and $\tau_{c}(X .(x)) \stackrel{x \rightarrow \infty}{\longrightarrow} \infty$ a.s.) and $f$ is increasing in $t$.

To establish uniqueness suppose that $\phi$ is a solution to (2.3.1); clearly, for any $T, \phi$ is bounded on $\mathbb{R}_{+} \times[0, T]$ (we extend the definition of $\phi$ in the obvious way: $\phi(x, t)=(c-x)$ for $x \leq b(t))$. Now look at

$$
M_{t}^{T}=e^{-\alpha(t \wedge \tau)} \phi\left(X_{t \wedge \tau}, T-(t \wedge \tau)\right)
$$

where

$$
\tau=\inf \left\{s \geq 0: X_{s}=b(s)\right\}
$$


and

$$
X_{0}=x \geq b(T) .
$$

It is immediate from Itô's formula and (2.3.1) that $M_{t}^{T}$ is a bounded martingale and so

$$
\begin{aligned}
M_{0}^{T} \equiv \phi(x, T)=\mathbb{E} M_{T}^{T} & =\mathbb{E} e^{-\alpha(\tau \wedge T)} g\left(X_{\tau \wedge T}\right) \\
& =f(x, T) \quad \forall T \in \mathbb{R}_{+}
\end{aligned}
$$

(since we know $\tau \wedge T$ is optimal for the $T$-horizon problem)

Proposition 2.3.2 The pair $(f, b)$ are a solution pair $(\phi, h)$ to the free boundary problem:

$$
\left.\begin{array}{l}
\mathcal{L} \phi=0: x>h(t) \\
\phi(h(t), t)=c-h(t) \\
\phi_{x}(h(t), t)=-1 \\
\phi(x, 0)=(c-x)^{+}: x \geq h(0) \\
\phi(x, t) \stackrel{x \rightarrow \infty}{\longrightarrow} 0 \text { uniformly on compact sets }
\end{array}\right\}
$$

and $f$ is maximal.

We shall need the following Proposition to prove Proposition 3.3.2.

Proposition 2.3.3 The function $f$ is $C^{1}$ in $x$.

Proof: Define

$$
M_{s} \equiv{ }^{t} M_{s}^{f} \stackrel{\text { def }}{=} e^{-\alpha s} f\left(X_{s}, t-s\right)+\alpha c \int_{0}^{s} e^{-\alpha u} I\left(X_{u} \leq h(t-u)\right) d u
$$

It follows from Theorem 5 and Corollary 6 of Jacka (1989) that, since $\partial D=\{(b(s), s) ; s \geq 0\}$ has zero (two-dimensional ) Lebesgue measure and the decreasing component of the semi-martingale decomposition of $\left(e^{-\alpha t} g\left(X_{t}\right)\right)$ is absolutely continuous with respect to Lebesgue measure, ${ }^{t} M_{s}^{f}$ is a martingale. Thus

$$
f(x, t)=M_{0}=\mathbb{E} M_{t}=K(x, t)+\alpha c \int_{0}^{t} e^{-\alpha u} \psi(x, b(t-u), u) d u
$$

where $\quad K(x, t)=\mathbb{E} e^{-\alpha t}\left(c-X_{t}(x)\right)^{+} \quad$ [the price of the European option]

$$
\psi(x, y, t)=\mathbb{P}\left(X_{t}(x) \leq y\right)
$$

From this representation it follows that

$$
f_{x}(x, t)=\alpha c \int_{0}^{t} e^{-\alpha s} \psi_{x}(x, b(t-s), s) d s \quad+\quad K_{x}(x, t) \quad \text { Lebesgue a.e. }
$$

Thus we need only establish that $r(x, t) \stackrel{\text { def }}{=} \int_{0}^{t} e^{-\alpha s} \psi_{x}(x, b(t-s), s) d s$ is continuous. Since $b$ is bounded and Borel-measurable it is sufficient to show that, for any Borel 
measurable $c, d$ given by

$$
d(x, t)=\int_{0}^{t} e^{-\alpha s} n\left(x-c(s), \sigma^{2} s\right) d s
$$

(where $n(x, v)$ is the density at $x$ of a normal random variable with mean zero and variance $v$ ), is continuous in $x$. If we note that $\left|n_{x}\right| \leq\left(\frac{e}{2 \pi}\right)^{1 / 2} /\left(\sigma^{2} t\right)$ and $0<n<$ $\frac{1}{\sigma(2 \pi t)^{1 / 2}}$ then we see that

$$
\begin{aligned}
|d(x, t)-d(y, t)| & \leq \int_{0}^{\delta} \frac{d s}{\sigma(2 \pi s)^{1 / 2}}+\int_{\delta}^{t}|x-y|\left(\frac{e}{2 \pi}\right)^{1 / 2} /\left(\sigma^{2} s\right) d s \\
& =\frac{2 \sqrt{\delta}}{\sigma \sqrt{2 \pi}}+|x-y|\left(\frac{e}{2 \pi}\right)^{1 / 2} \ln (t / \delta) / \sigma^{2} .
\end{aligned}
$$

Thus, setting $\delta=(x-y)^{2}$, we see that

$$
|d(x, t)-d(y, t)| \leq K_{t}|x-y|(1+|\ln | x-y||)
$$

so that $d$ is locally Hölder continuous.

Proof of Proposition 2.3.2: The fact that $(f, b)$ satisfy $(2.3 .2)$ follows immediately from Propositions 2.3.1 and 2.3.3.

To show that $f$ is maximal; suppose that $(\phi, h)$ is a solution pair to $(2.3 .2)$ then by the same argument as that used in the proof of Proposition 2.3.1

$$
\phi(x, t) \leq \mathbb{E} e^{-\alpha\left(\tau_{h} \wedge t\right)} g\left(X_{\tau_{h} \wedge t}\right) \quad(\text { since } g(x) \geq c-x)
$$

where

$$
\tau_{h}=\inf \left\{s \geq 0: X_{s}=h(t-s)\right\}
$$

thus

$$
\phi(x, t) \leq f(x, t)=\sup _{\tau \leq t} \mathbb{E} e^{-\alpha \tau} g\left(X_{\tau}\right)
$$

2.4 We return now to the proof of the continuity of $b$.

Proof of Proposition 2.2.4: Recall that $\mathcal{L} f=0$ in $D$ so that, since $\frac{\partial f}{\partial t} \geq 0$ and $\frac{\partial f}{\partial x} \leq 0$,

$$
1 / 2 \sigma^{2} x^{2} \frac{\partial^{2} f}{\partial x^{2}} \geq \alpha f
$$

in $D$. Thus, defining $D^{n} \stackrel{\text { def }}{=} D \cap[0, c] \times\left[n^{-1}, n\right]$,

$$
\inf _{(x, t) \in D^{n}} 1 / 2 \sigma^{2} x^{2} \frac{\partial^{2} f}{\partial x^{2}} \geq \epsilon_{n}>0,
$$

since $f>0$ in $\operatorname{cl}\left(D^{n}\right)$ and is continuous, while $\operatorname{cl}\left(D^{n}\right)$ is compact. 
Now, given $t>0$ take $N: t \geq N^{-1}$, then for any $s$ with $N \geq s>t$ and any $x \in[b(t+)+\eta, c]:$

$$
\begin{aligned}
f(x, s)-g(x, s) & =\int_{b(s)}^{x} 1 / 2 \sigma^{2} x^{2} \frac{\partial^{2} f}{\partial x^{2}}(u, s)-1 / 2 \sigma^{2} x^{2} \frac{\partial^{2} g}{\partial x^{2}}(u, s) d u \\
& \geq \eta \epsilon_{n},
\end{aligned}
$$

since $1 / 2 \sigma^{2} x^{2} \frac{\partial^{2} g}{\partial x^{2}}$ vanishes on $[0, c] \times \mathbb{R}_{+}, f$ and $g$ agree on $b$ up to first derivatives and $b$ is decreasing. Thus, taking a sequence $\left(s_{n}\right)$ decreasing strictly to $t$, it follows from the continuity of $f-g$ that

$$
f(b(t+)+\eta, t)-g(b(t+)+\eta, t) \geq \eta \epsilon_{N}>0,
$$

and hence that $(b(t+)+\eta) \in D_{t}$ for all $\eta>0$, implying that $b(t+) \geq b(t)$. Rightcontinuity of $b$ now follows from the fact that $b$ is decreasing

Remark The representation (2.3.3) is similar to one obtained, in a more general context, in El Karoui and Karatzas (1989). We thank an anonymous referee for pointing out this reference.

\section{$\S 3$ Pricing the option}

We now show the connection between the optimal stopping problem of section 2 and the option pricing problem.

We are confronted with the problem of pricing an option to sell, at any time before $T$, one unit of the underlying stock at a fixed price $c$ (an American put option). Harrison and Pliska (1981) have shown how to solve this, and many other, problems when the option is European, i.e. the right to sell may only be exercised at time $T$. Bensoussan (1984) and Karatzas (1987) have generalised their arguments to the case of American options but for the sake of completeness we shall run through the argument here.

We assume that the underlying stock-price is given by

$$
d X_{t}=\sigma X_{t} d B_{t}+\mu X_{t} d t
$$

where $B$ is a Brownian motion, and that interest on cash holdings is paid at a fixed rate $\alpha$, and that the stock generates no dividends.

Theorem 3.1 Let

$$
d X_{t}^{\prime}=\sigma X_{t}^{\prime} d B_{t}+\alpha X_{t}^{\prime} d t
$$

with

$$
X_{0}^{\prime}=X_{0}
$$

and let

$$
f(x, t)=\sup _{\tau \leq t} \mathbb{E}_{x} e^{-\alpha \tau}\left(c-X_{\tau}^{\prime}\right)^{+} \quad \text { as in section } 2
$$


be the optimal payoff from the problem of optimally stopping the process $e^{-\alpha s}\left(c-X_{s}^{\prime}\right)^{+}$. Then there is a self-financing portfolio of stock and cash, $\left(\left(Y_{s}, Z_{s}\right) ; 0 \leq s \leq t\right)$, with $Y$ and $Z$ adapted, whose value at time $s, V_{s}$, satisfies

$$
V_{s} \equiv\left(X_{s} Y_{s}+Z_{s}\right) \geq\left(c-X_{s}\right)^{+}
$$

and such that if $\tau$ is the optimal stopping time corresponding to the payoff $f\left(X_{0}, t\right)$ then

$$
V_{\tau}=\left(c-X_{\tau}\right)^{+}
$$

so that $X_{0} Y_{0}+Z_{0} \equiv f\left(X_{0}, t\right)$ is the fair price for the option. $\dagger$

Proof: we have shown in section 2 that the optimal policy for the stopping problem with horizon $t$ is to exercise when $X_{s}^{\prime}$ falls below $b(t-s)$, and that moreover $f$ is piecewise $C^{2}$ in $x, C^{1}$ in $t$, with

$$
d\left(e^{-\alpha s} f\left(X_{s}^{\prime}, t-s\right)\right)=e^{-\alpha s} f_{x}\left(X_{s}^{\prime}, t-s\right) \sigma X_{s}^{\prime} d B_{s}-\alpha c e^{-\alpha s} I\left(X_{s}^{\prime} \leq b(t-s)\right) d s
$$

so that

$$
d f\left(X_{s}^{\prime}, t-s\right)=\alpha f\left(X_{s}^{\prime}, t-s\right)+f_{x}\left(X_{s}^{\prime}, t-s\right) \sigma X_{s}^{\prime} d B_{s}-\alpha c I\left(X_{s}^{\prime} \leq b(t-s)\right) .
$$

Setting $Y_{s}=f_{x}\left(X_{s}, t-s\right)$

$$
\begin{array}{ll}
Z_{s}=f\left(X_{s}, t-s\right)-X_{s} f_{x}\left(X_{s}, t-s\right)+e^{\alpha s} \alpha c \int_{0}^{s} e^{-\alpha u} I\left(X_{u} \leq b(t-u)\right) d u \\
\text { and } & V_{s} \equiv X_{s} Y_{s}+Z_{s}=f\left(X_{s}, t-s\right)+\alpha c e^{\alpha s} \int_{0}^{s} e^{-\alpha u} I\left(X_{u} \leq b(t-u)\right) d u(2.1)
\end{array}
$$

we see that

$$
\begin{aligned}
d V_{s}= & \alpha f\left(X_{s}, t-s\right) d s+\sigma f x\left(X_{s}, t-s\right) X_{s} d B_{s} \\
& +(\mu-\alpha) f_{x}\left(X_{s}, t-s\right) X_{s} d s+\alpha\left(e^{\alpha s} \alpha c \int_{0}^{s} e^{-\alpha u} I\left(X_{u} \leq b(t-u) d u\right) d s\right. \\
= & Y_{s} d X_{s}+\alpha Z_{s} d s
\end{aligned}
$$

so that $V$ is the value of a self-financing portfolio (since $Y_{s}, X_{s}$, and $Z_{s}$ are continuous so $d V_{s}=Y_{s-} d X_{s}+\alpha Z_{s-} d X_{s}$ which is the 'instantaneous return' on a portfolio of $Y_{s-}$ shares and $Z_{s-}$ cash); whilst, from $(2.1), V_{t} \geq\left(c-X_{t}\right)^{+}$since $f(x, t) \geq(c-x)^{+}$.

\section{$\S 4$ An integral transform of the stopping boundary}

4.1 We first establish a martingale result.

$\dagger$ As is demonstrated in the proof, if someone writes the option and sells it for $V_{0}$ and then purchases the portfolio $\left(X_{0}, Y_{0}\right)$ and operates it in the prescribed manner then they will not lose money and will make money unless (essentially) the purchaser uses the option optimally - this is what we mean by $V_{0}$ being the fair price for the option. 
Theorem 4.1.1: Let $(\phi, h)$ be a solution pair to the free-boundary problem $(2.3 .2)$; then, extending the definition of $\phi$ in the obvious way and defining

$$
M_{s} \equiv{ }^{t} M_{s}^{\phi} \stackrel{\text { def }}{=} e^{-\alpha s} \phi\left(X_{s}, t-s\right)+\alpha c \int_{0}^{s} e^{-\alpha u} I\left(X_{u} \leq h(t-u)\right) d u
$$

$\left(M_{s}: 0 \leq s \leq t\right)$ is a martingale.

Proof: From Itô's formula,

$$
\begin{aligned}
d M_{s}= & e^{-\alpha s}(\mathcal{L} \phi)\left(X_{s}, t-s\right) d s+\alpha c e^{-\alpha s} I\left(X_{s} \leq h(t-s)\right) d s \\
& +e^{-\alpha s} \phi_{x}\left(X_{s}, t-s\right) \sigma X_{s} d B_{s},
\end{aligned}
$$

since $\phi$ is $C^{1}$ and piecewise $C^{2}$ in $x$, and piecewise $C^{1}$ in $t$, but

$$
(\mathcal{L} \phi)(x, s)= \begin{cases}0 & : x>h(s) \\ -\alpha c & : x<h(s)\end{cases}
$$

so $M$ is a local martingale. Since $\phi(x, u) \rightarrow 0$, uniformly for $u$ in a compact set, as $x \rightarrow \infty$, and $\phi$ is $C^{1}$ in $x, \phi$ is bounded on $\mathbb{R}_{+} \times[0, t]$ so $M$ is, in fact, a martingale.

4.2 We may now show that $(f, b)$ are essentially the unique solution pair to (2.3.2):

Theorem 4.2.1: The pair $(f, b)$ are the unique solution pair $(\phi, h)$ (with $h$ Lebesguemeasurable) to (2.3.2) satisfying $h(t)>0$ infinitely often.

Proof: We have already established in Proposition 3.3.2 that $(f, b)$ satisfy $(2.3 .2)$. Now suppose $(\phi, h)$ are another solution pair to $(2.3 .2)$ with $h(t)>0$, then define $M_{s}^{0}(x)$ and $M_{s}^{1}(x)$ by

$$
\begin{aligned}
& M_{s}^{0}=e^{-\alpha s} f\left(X_{s}(x), t-s\right)+\alpha c \int_{0}^{s} e^{-\alpha u} I\left(X_{u}(x) \leq b(t-u)\right) d u \\
& M_{s}^{1}=e^{-\alpha s} \phi\left(X_{s}(x), t-s\right)+\alpha c \int_{0}^{s} e^{-\alpha u} I\left(X_{u}(x) \leq h(t-u)\right) d u
\end{aligned}
$$

Now fix $t>0$ and consider

$$
\begin{gathered}
x<b(\infty) \wedge h(t) \\
\leq b(t) \wedge h(t)
\end{gathered}
$$

Since $x<b(t) \wedge h(t)$,

$$
f(x, t)=\phi(x, t)=g(x),
$$

and so, defining $M=M^{0}-M^{1}, M_{0}=0$. Now define

$$
\tau=\inf \left\{s \geq 0: X_{u}(x)>b(t-u)\right\} \wedge t
$$


then $\mathbb{E} M_{\tau \wedge s}=M_{0}=0$ but

$$
\begin{aligned}
M_{\tau \wedge s}= & e^{-\alpha \tau \wedge s}\left(f\left(X_{\tau \wedge s}(x), t-\tau \wedge s\right)-\phi\left(X_{\tau \wedge s}, t-\tau \wedge s\right)\right) \\
& + \\
& \alpha c \int_{0}^{\tau \wedge s} e^{-\alpha u} I\left(X_{u}(x)>h(t-u)\right) d u
\end{aligned}
$$

(since, on $[0, \tau) X_{u}(x) \leq b(t-u)$ ), and since each term on the right hand side of (4.2.1) is non-negative (since $f$ is maximal) we conclude that

$$
X \cdot \wedge \tau \wedge s(x) \leq h(t-\cdot \wedge \tau)(\text { Lebesgue } \times \mathbb{P} \text { a.e. })
$$

and

$$
\begin{aligned}
\phi\left(X_{s \wedge \tau}(x), t-s\right) & =f\left(X_{s \wedge \tau}(x), t-s\right) \\
& =g\left(X_{s \wedge \tau}(x)\right)(\mathbb{P} a . s .)
\end{aligned}
$$

so that we may conclude that $h(s) \geq b(s)$ Lebesgue a.e. $(s \leq t)$.

However, if we consider $M_{t}$ :

$$
M_{t}=c \alpha \int_{0}^{t} e^{-\alpha s}\left\{I\left(X_{s}(x) \leq b(t-s)\right)-I\left(X_{s}(x) \leq h(t-s)\right)\right\} d s
$$

(because $\phi(x, 0)=f(x, 0)=g(x))$, we see that the integrand is non-positive but $\mathbb{E} M_{t}=$ $M_{0}=0$ so that the integrand is (Lebesgue $\times \mathbb{P}$ )a.e. zero: we may now conclude that $b=h$ lebesgue a.e. (on $[0, t])$. Finally (taking an arbitrary $x$ and $s \leq t$ )

$$
\phi(x, s)={ }^{s} M_{0}^{1}=\mathbb{E}^{s} M_{s}^{1}=\mathbb{E}^{s} M_{s}^{0}={ }^{s} M_{0}^{0}=f(x, s)
$$

so that $\phi=f$ and hence $h \leq b$ everywhere on $[0 \leq s \leq t]$ (since $f>g$ on $D$ ); this implies that $h=b$ since $\mathcal{L} f \neq 0$ on $D^{c}$ Note that the argument fails if $\exists t: h(s) \equiv 0, \forall s \geq t$.

Remark: A close analysis shows that the case where $h(s)=0$ for all $s>t$ corresponds to the case of prohibited stopping before horizon $t$.

Theorem 4.2.2: The boundary $b(\cdot)$ is the unique left-continuous solution $h(\cdot)$ satisfying $c>h>0 \quad \forall t>0$ of the integral equation:

$$
(c-x)=K(x, t)+\alpha c \int_{0}^{t} e^{-\alpha s} \psi(x, h(t-s) ; s) d s \quad \forall x \leq h(t)
$$

where, as before

$$
\begin{aligned}
& K(x, t)=\mathbb{E} e^{-\alpha t}\left(c-X_{t}(x)\right)^{+} \quad \text { [the price of the European option] } \\
& \psi(x, y, t)=\mathbb{P}\left(X_{t}(x) \leq y\right)
\end{aligned}
$$

Proof: the fact that $b$ satisfies (4.2.2) follows immediately from the fact that

$$
\left(M_{s}^{0}: s \leq t\right) \equiv e^{-\alpha s} f\left(X_{s}(x), t-s\right)+\alpha c \int_{0}^{s} e^{-\alpha u} I\left(X_{u}(x) \leq b(t-u)\right) d u
$$


is a martingale, started at $c-x$, if $x \leq b(t)$.

If we now suppose that $h$ solves (4.2.2) and define

$$
\phi(x, t)=K(x, t)+\alpha c \int_{0}^{t} e^{-\alpha s} \psi(x, h(t-s), s) d s
$$

then it is clear that

$$
M_{s}^{1} \equiv e^{-\alpha s} \phi\left(X_{s}(x), t-s\right)+\alpha c \int_{0}^{s} e^{-\alpha u} I\left(X_{u}(x) \leq h(t-u)\right) d u
$$

is a $\left(\sigma\left(B_{u}: u \leq s\right) ; s \leq t\right)$ martingale for every choice of $(x, t) \in \mathbb{R}_{+}^{2}$. The proof now follows along the same lines as that of Theorem 4.2.1. Firstly note that, defining

$$
\tau=\inf \left\{s \geq 0: X_{s}(x) \leq h(t-s) \wedge t\right\}
$$

we see that for $x>h(t)$ :

$$
\begin{aligned}
\phi(x, t) \equiv M_{0}^{1} & =\mathbb{E} e^{-\alpha \tau} \phi\left(X_{\tau}, t-\tau\right) \\
& =\mathbb{E} e^{-\alpha \tau} g\left(X_{\tau}\right)
\end{aligned}
$$

so that for $x \geq h(t), \phi \leq f$, whilst, from (4.2.2), $\phi=g \leq f$ for $x \leq h(t)$.

Secondly we see, on taking $0<x \leq h(t) \wedge b(t)$ and setting

$$
\tau \prime=\inf \left\{s \geq 0: X_{s}(x) \geq b(t-s)\right\} \wedge t,
$$

that

$$
\begin{aligned}
0=f(x, t)-\phi(x, t)= & \mathbb{E} e^{-\alpha \tau \prime}\left(f\left(X_{\tau^{\prime}}, t-\tau \prime\right)-\phi\left(X_{\tau^{\prime}}, t-\tau \prime\right)\right) \\
& + \\
& \mathbb{E} \alpha c \int_{0}^{\tau \prime} e^{-\alpha u} I\left(X_{u}>h(t-u)\right) d u
\end{aligned}
$$

and, since both the terms on the right of (4.2.3) are non-negative we conclude that $h \geq b$ (Lebesgue a.e.), but this implies that $\phi \geq f$, so $\phi=f$ and so (by left continuity) $h=b$ 
Acknowledgements I would like to thank the following people for helpful discussions on both the theoretical and the practical aspects of option-pricing: Andrew Carverhill, Mark Eban, Nick Webber, and Marc Yor.

\section{References}

Bensoussan,A (1984): On the theory of option pricing Acta Applicandae Mathematicae 2,139-158.

Black, F and Scholes, M (1973): The pricing of options and corporate liabilities. J. Political Econ. 81,637-659.

Carr, P, Jarrow, R, and Myneni, R (1990): Alternative characterisations of American put options. Preprint: Cornell University

El Karoui,N and Karatzas, I (1989): A new approach to the Skorohod problem and its applications. Preprint: Columbia University

Friedman,A (1975): Stochastic Differential Equations and Applications I. Academic Press, New York.

Harrison, J M and Pliska,S R (1981): Martingales and stochastic integrals in the theory of continuous trading. Stoch. Proc. and Appl. 11,215-260.

Jacka, S D (1989): Local times, optimal stopping and semi-martingales.Preprint: Warwick University

Jacka, S D and Lynn, J R (1990): Finite-horizon optimal stopping, obstacle problems and the shape of the continuation region. Preprint: Warwick University

Karatzas, I (1988): On the pricing of American options. Applied Maths $\& 5$ Optimization 17,37-60.

Kim, I J (1990): The analytic valuation of American options. Preprint: New York University

Krylov, N V (1980): Controlled Diffusion Processes. Springer, New York.

McKean, H P (1965): A free boundary problem for the heat equation arising from a problem in Mathematical Economics. Industrial Management Review 6, 32-39.

Van Moerbeke, P (1976): On optimal stopping and free boundary problems. Arch. Rational Mech. and Analysis 60, 101-148. 